\title{
Die 62. Jahrestagung der DGP 2020 in Leipzig - Eine Internationale Konferenz
}

Tobias Hirsch

Die Jahrestagung der DGP fand in diesem Jahr vom 2.-5. September 2020 in Leipzig statt.

Ihre Vorbereitung war geprägt von turbulenten Entwicklungen. Beginnend damit, dass die Vorbereitungszeit für eine so große Konferenz mit insgesamt weniger als 2 Jahren sehr kurz war, hat in der Schlussphase der Organisation die Corona-Pandemie für erhebliche Spannung und Verunsicherung gesorgt.

Unter diesen Rahmenbedingungen geriet die Umsetzung des Mottos „Phlebologie national und international" zu einer besonderen Herausforderung.

Um unsere Jahrestagung als internationale Konferenz zu gestalten, mussten 3 Grundlagen geschaffen werden. Renommierte internationale Experten mussten als Referenten gewonnen werden, um die Tagung für unsere treuen Teilnehmer zu bereichern. Das Interesse eines internationalen Publikums für die deutsche Phlebologie durfte selbstbewusst erwartet werden, aber es musste die Sprachbarriere überwunden werden, um das Programm sowohl für die internationalen Gäste als auch für unsere einheimischen Kollegen attraktiv zu machen. Letztlich musste die Tagung auch wirkungsvoll beworben werden. Darüber hinaus war es erforderlich, die für das ehrgeizige Projekt erforderlichen zusätzlichen Mittel einzuwerben.

Bestärkt von der großen Zustimmung der Mitglieder der DGP und des Vorstandes wurde das Projekt DGP goes international angegangen.

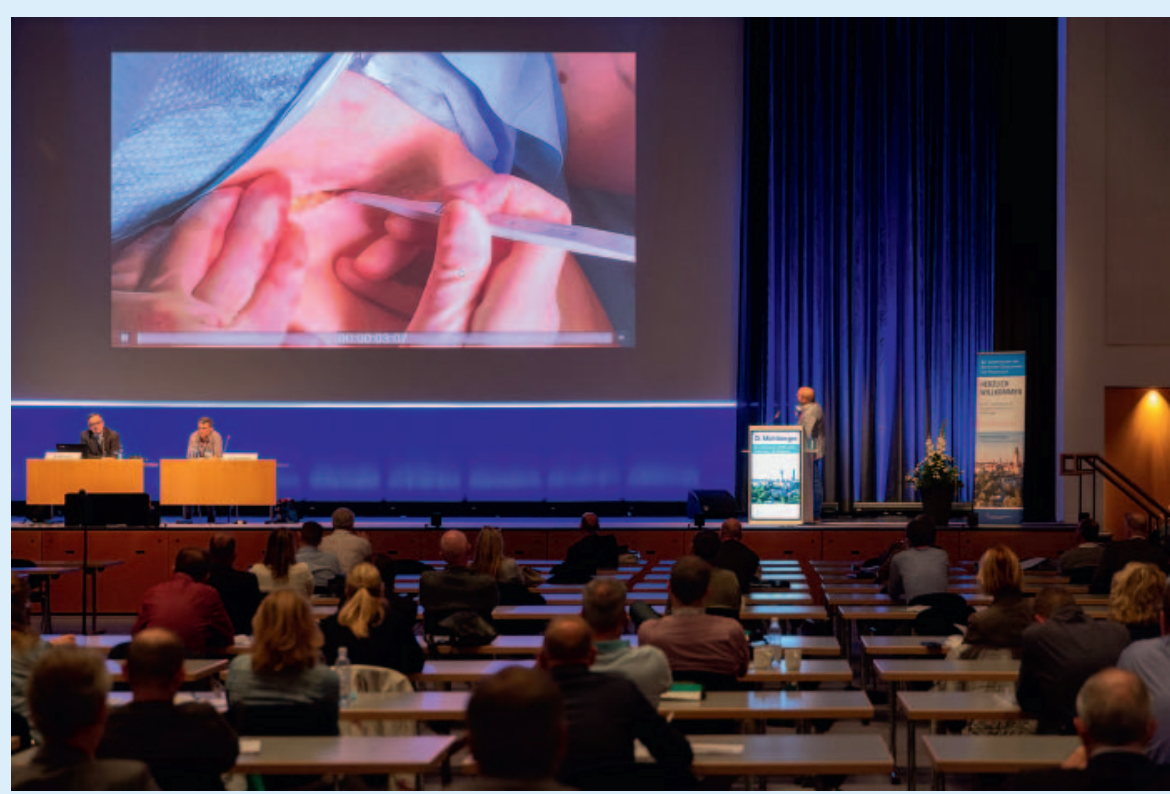

Case of the day: Großzügige Räume erlaubten die Diskussion der vorgestellten Fälle unter Wahrung des Abstandsgebotes.

Dass es nun trotz COVID-19 erfolgreich realisiert und zu einem Höhepunkt der internationalen Gefäßmedizin des Jahres 2020 werden konnte, ist der Tatsache zu verdanken, dass die DGP verlässliche Partner hat: unsere Kongressagentur wikonect, unsere Partner aus der Industrie und die einzelnen Mitglieder der Fachgesellschaft selbst, die ihre Kreativität, Expertise und Idealismus einbringen und damit maßgeblich seit Jahrzehnten das hohe Niveau und die einzigartige Atmosphäre unserer Jahrestagungen prägen.

\section{Internationale Gäste und Educational Grant}

Die deutsche Phlebologie genießt seit vielen Jahren einen exzellenten Ruf. Umso verwunderlicher ist es eigentlich, dass sie in der letzten Zeit auf dem internationalen Parkett etwas unterrepräsentiert erschien. Diese Situation zu ändern, war das Ziel von Leipzig 2020.

Dank zahlreicher persönlicher Kontakte waren in der Faculty unter den insgesamt 162 Referenten 36 internationale Experten u. a. aus unseren unmittelbaren europäischen Nachbarländern Frankreich (5), Polen (5), der Schweiz (3), Österreich (2), Tschechien (1) und den Niederlanden (1). Gerade die Intensivierung der Beziehungen zu den Fachgesellschaften unserer unmittelbaren Nachbarn lag mir als Kongresspräsident dabei besonders am Herzen. Weitere europäische Sprecher kamen aus Großbritannien (3), Italien (3), Russland (2), Spanien (1) und Lettland (1). Darüber hinaus sind namhafte Phlebologen aus den USA (4), Argentinien (2), der Türkei (2) 


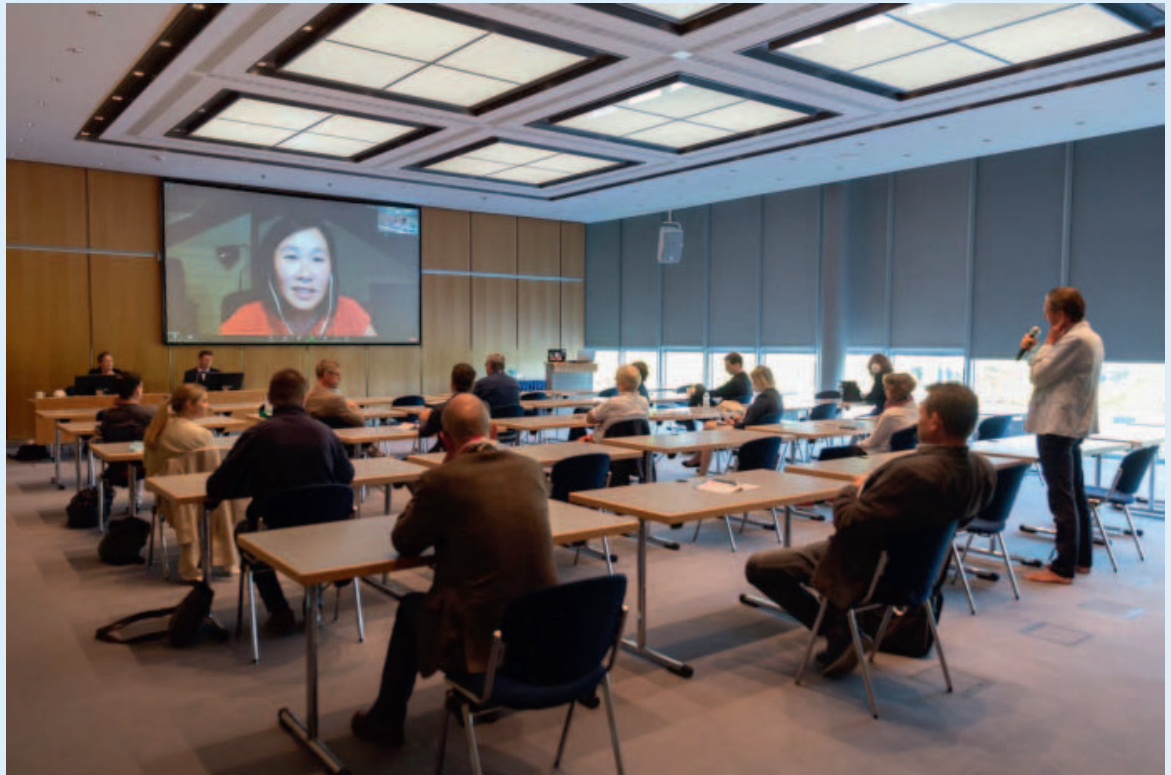

Ask the expert - Sclerotherapy: Teilnehmer der Sitzung konnten mit Margaret Mann diskutieren, die per Zoom aus den USA zugeschaltet war.

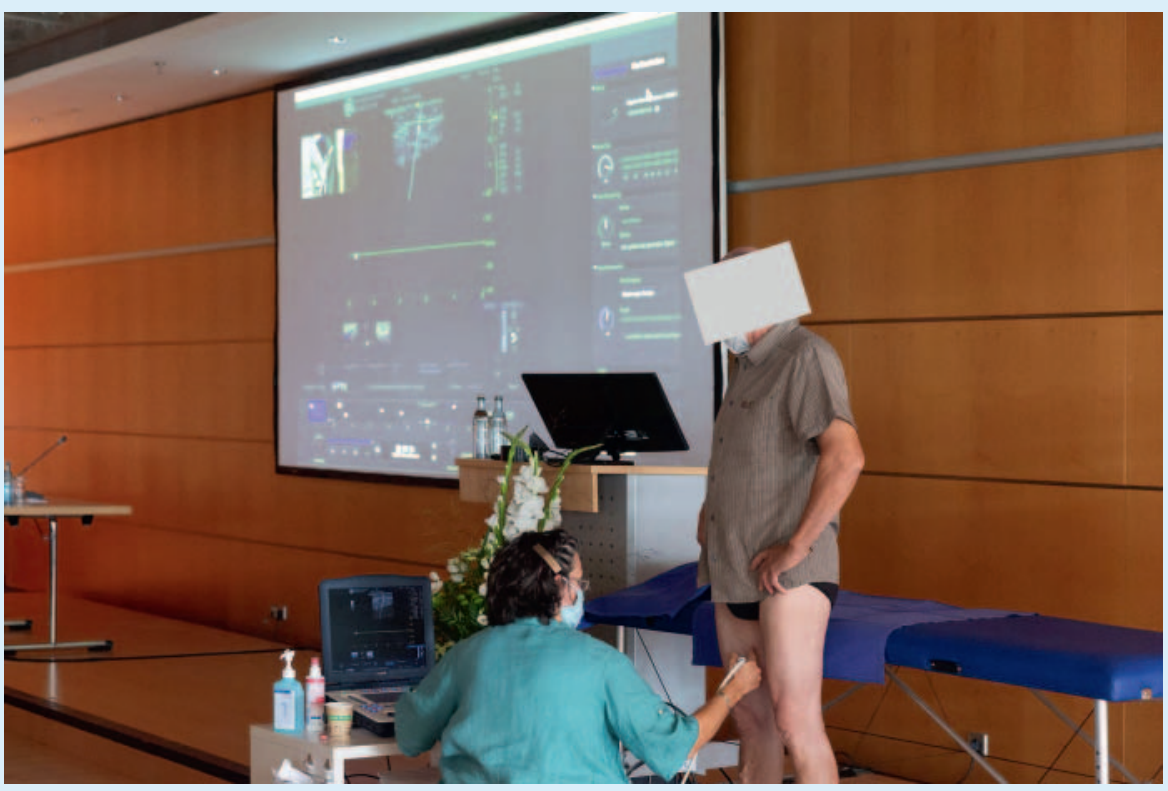

Ultrasound Masterclass: Dr. Mendoza vermittelt live Tipps und Tricks für die Refluxdiagnostik.

und Australien (1) unserer Einladung gefolgt. Diese erstklassige Besetzung ermöglichte es, gemeinsam mit unseren hervorragenden deutschen Experten von Donnerstag bis Samstag einen kompletten internationalen Block anzubieten.

Während am Kongress-Donnerstag die ganztätige Ultrasound Masterclass von Frau Dr. Erika Mendoza mit über 80 internationalen Teilnehmern auf Englisch präsen-
Das Programm wurde im Vorfeld intensiv international beworben. Dabei erhielten wir Unterstützung befreundeter Fachgesellschaften wie etwa der französischen und der polnischen, die uns bereits sehr früh versicherten, dass sie großes Interesse an einer Zusammenarbeit mit der DGP haben, ebenso über den Verteiler des European Venous Forum. Darüber hinaus wurde regelmäßig in einschlägigen Internetforen wie der webbasierten Zeitschrift Venous News und WhatsApp-Gruppen über die Tagung informiert, und natürlich, was ganz besonders wichtig war, persönlich auf zahlreichen internationalen Konferenzen.

Mit Spannung durfte erwartet werden, inwieweit das Programm auch wirklich internationale Gäste anziehen sollte.

Um das Programm auch für den internationalen phlebologischen Nachwuchs interessant zu machen, konnten wir mit der finanziellen Unterstützung unserer Partner aus der Industrie ein Educational Grant für 19 ausländische Nachwuchsphlebologen ausloben, welches die Kongressgebühr sowie die Kosten für die Ultrasound Masterclass komplett deckte. Die Firmen, denen an dieser Stelle besonders zu danken ist, waren Kreussler, Sigvaris, Bauerfeind und Biolitec.

Mit großer Freude durften wir unter den insgesamt 946 Teilnehmern 113 Gäste aus 27 verschiedenen Ländern von Nepal bis Brasilien und von Australien bis Russland begrüßen.

\section{COVID-19-Pandemie - Zeit für Neue Veranstaltungsformate}

Angesichts dieser spektakulären Zahlen muss erneut auf die exzellente organisatorische Umsetzung des Kongresskonzeptes durch wikonect verwiesen werden. Die Durchführung eines Kongresses dieser Größenordnung und noch dazu mit internationaler Beteiligung unter den Bedingungen der COVID-19-Pandemie verlangte nach weiteren besonderen Maßnahmen. Die Tagung war ausdrücklich als Präsenzveranstaltung vorgesehen. Das machte die Realisierung eines strengen Hygienekonzeptes erforderlich, welches gemeinsam mit dem 

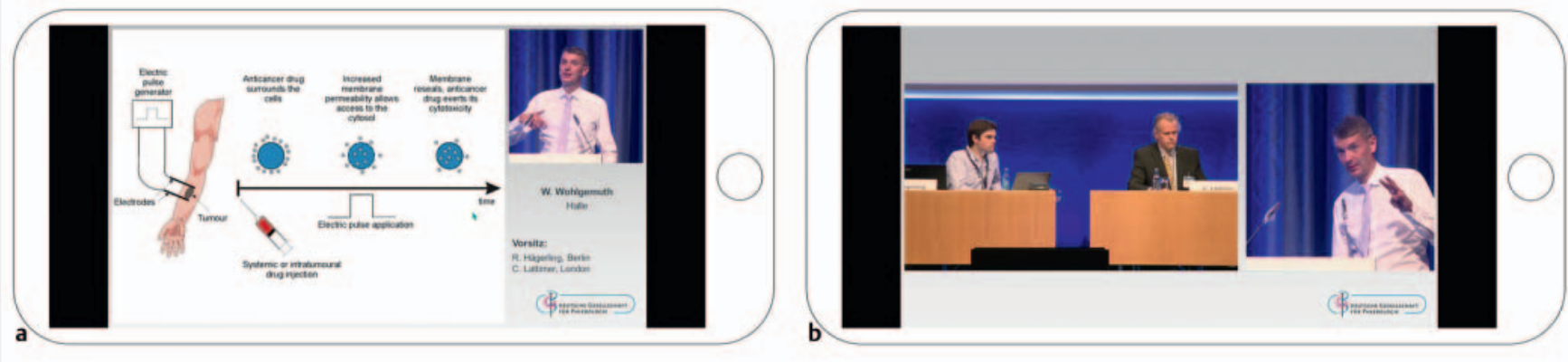

Livestream auf dem Handy-Display: Virtuell meets live.

Gastgeber, der Messe Leipzig, erarbeitet wurde. Als ausgesprochener Glücksfall erwies sich aufgrund seiner Architektur und Größe das Congress Center Leipzig (CCL). Die Vielzahl der großen Säle und kleineren Räume ließ neben den beiden Hauptsitzungen auch die Durchführung bewährter Formate wie Ask-the-Expert und Hands-onKurse unter Einhaltung des gebotenen Sicherheitsabstandes zu. Kleinere Formate, wie die Workshops Liposuktion, endovenöse Verfahren oder Sklerotherapie, konnten aufgrund des Hygienekonzeptes stattfinden und waren sehr gut besucht.

Nach Monaten der Abstinenz von LiveEvents war vom ersten Ton der Bourrée von Bach in e-Moll zur Eröffnungsveranstaltung bis zum Gesellschaftsabend die große Freude aller an der Begegnung, dem persönlichen Austausch und der physischen Präsenz spürbar.

Gerade für ausländische Gäste, aber auch für viele deutsche Kollegen standen Reisebeschränkungen einer persönlichen Teilnahme an unserer Jahrestagung im Weg. Eigens für diesen Zweck wurde ein multimediales Konzept entwickelt, was sich maßgeblich von den in den vergangenen
Monaten in aller Welt praktizierten Webinaren abheben sollte. Referenten, die selbst nicht anreisen durften, konnten per Zoom auftreten und sich der Diskussion stellen. Teilnehmer konnten per Live-Stream virtuell in den Sitzungssälen an den beiden Hauptsitzungen teilnehmen und per ChatFunktion mit dem Panel und den Rednern kommunizieren.

180 der Kongressteilnehmer und 26 der Referenten ermöglichte dieses Hybridformat einen virtuellen Tagungsbesuch. Ich darf mit Begeisterung erwähnen, dass die technische Umsetzung hervorragend war und durchaus internationales Aufsehen erregt hat. So war doch die 62. Jahrestagung der DGP 2020 in Leipzig die erste internationale gefäßmedizinische Konferenz seit dem Ausbruch der Pandemie.

Als Kongresspräsident möchte ich mich noch einmal bei allen bedanken, die ihren Anteil am Gelingen von „Phlebologie - national und international“ hatten, insbesondere bei allen deutschen und internationaIen Teilnehmern, die der Veranstaltung Leben und Atmosphäre verliehen haben.

Tobias Hirsch

\section{Internationales Feedback:}

"German Society of Phlebology stage the world's first hybrid \#medicalconference combining a physical venue and comprehensive online program streamed live from the \#Leipzig epicentre....

It was indeed a tremendous honour to have been engaged with this \#globalevent."

Joseph Gracé, Sydney/Australien via LinkedIn

"Job well done. It was a lovely conference, would have stayed longer if it wasn't the time difference. Fantastic show ... I hope we have similar turn out, will see."

Margaret Mann, Hendersonville/USA, Congress Chair AVLS Oct 2020

“Thank you Tobias! And congratulations this was a really international and (saying this as participant) great and interesting congress. All worked perfectly."

Tomasz Urbanek, Kattowitz/Polen

"Thank you for your invitation and excellent congress programme. I am sorry I could not be there in person..."

Manjit Gohel, Cambridge/Großbritannien 